position. Both Lawford and I had grave doubts as to the expediency of this, and Holmes Spicer had also pronounced in favour of no interference. However, the father, who is a medical man, wished Landolt's suggestion to be tried, and asked me to do the operation. Under the anæsthetic, I found, as I had expected, that the visual axes were parallel, if not slightly divergent, and of course I had to aim at producing by advancement a divergence of approximately $15^{\circ}$, in which I was fairly successful.

In brief, her subsequent history is that her eyes vary from a condition of divergence, with very little spasm, to wild convergence with spasm of $9 \mathrm{D}$. or $10 \mathrm{D}$. She will go on comfortably using the right eye for some weeks without getting any spasm, but getting it at once on attempting to use the left eye, and then suddenly will find that the position is reversed, and that now she can only use the left eye. Despite this remarkable ocular history, she has been able to work for the last two years fairly steadily at the Ministry of Munitions, where she is still working.

Since the war began I have seen somewhat similar cases, to one of which I may refer briefly. Private $\mathrm{C}$. J. had a slight concussion of the brain in February, 1915. I saw him in July, 1915, with marked spasm of convergence, blepharospasm and great limitation of visual fields. His refraction without atropin was $-6 \mathrm{D}$. Sph. in each eye. Under full atropin he proved to be hypermetropic $+1 \mathrm{D}$. Sph. in each eye. The blepharospasm and convergence spasm gradually diminished without the accommodative spasm being much affected, but by February, 1916, all trace of spasm had gone, his visual fields were full, and his visual acuity was $6 / 6$ in each eye without any glasses.

I have put these two cases on record as examples of true spasm of accommodation, and I think that in each one is justified in adding the term "functional." Certainly the second case was typically a functional disturbance, and though the first case presents many complexities, I have little doubt that it, too, is best grouped under the same heading.

\title{
DISEASES OF THE EYE OF OBSCURE ORIGIN*
}

BY

D. Leighton Davies, M.D., M.S., F.R.C.S., ASSISTANT OPHTHALMIC SURGEON KING EDWARD VII HOSPITAL, CARDIFF.

THERE must be few ophthalmic surgeons to-day who are satisfied with having labelled a case as "rheumatic iritis." It is a comforting diagnosis from the patient's point of view, because the lay public

*A paper read before the Uphthalmological Society, May, 1917. The full communication will be published in the Society's Transactions. 
think they know all about "rheumatism," and the patient will therefore go away quite satisfied that the cause of the trouble has been probed. But although the term "rheumatic iritis" is a useful one, it should not blind us to the fact that we have not arrived at the ultimate cause of the condition when we use this term. Again, the term "gouty iritis" is a very loose one and conjures up but a vague mental picture of the pathogenesis of the process. Probably true gouty iritis may have existed at a time when acute gout was a prevalent disease amongst the middle-aged and elderly, but acute gout is, I believe, very uncommon nowadays, so uncommon indeed, as to be almost a rarity.

Acute inflammatory diseases of the uveal tract are, apart from injuries, probably almost invariably of infective origin, but it is not always so easy to prove this statement as to make it. The infection may be part of some other general infective disease, i.e., syphilis, tuberculosis, typhoid fever, and so on ; or, it may be the result of an auto-intoxication arising from various regions of the body. These may be :-

(1). The alimentary system.-Pyorrhœa alveolaris is recognized as a fertile source of infective process. The tonsils, again, when the seat of chronic infection, may be the cause of an iritis (as has, I believe, been shown). Lower down the tract intestinal fermentation has been shown to be a cause of irido-cyclitis. This was ably demonstrated by J. B. Lawford and others in the debate on alimentary toxæmia at the Royal Society of Medicine in 1913.

(2). The respiratory passages.-(a) The accessory nasal sinuses. I have seen a case where a constantly relapsing iritis was cured by operative treatment for pansinusitis which had its origin in a frontal sinus suppuration. The patient had refused to undergo the radical cure of her nasal suppuration for many years, during which time she had frequently recurring attacks of irido-cyclitis which brought her sight down to 6/60 in each eye. Since operation, now two years ago, she has had no relapses, though, owing to vitreous opacities and old deposits on the lens capsule, vision does not improve.*

(b) A chronic empyema or bronchiectasis may equally well produce the condition, though I have not come across such a case.

(3). The genito-urinary tract.- Under this head gonorrhœa may be accounted the most important factor. In the male a long forgotten attack of gonorrhœa may be the cause of an obstinately recurring iritis. I have notes of a man who has had slight attacks of iritis (about twice a year) for many years. He owned to a mild attack of gonorrhœa twenty years previously; after massage of the prostate, prostatic plugs and gonococci were found in the urine.

* Since writing this paper this patient has reported herself. She has been acting as an orderly in a military camp in North Wales, and has been " roughing " it during the whole of the severe weather of this last winter, without any relapse. Surely a proof that " cold" and "rheumatism" have nothing to do with her irido-cyclitis. 
Vaccine treatment decreased the frequency of the attacks, but did not cure him. Probably re-infection takes place from the prostatic crypts from whence it is almost impossible to expel the gonococci. In women, proof is not so easily obtained, but undoubtedly this is a factor that must ever be borne in mind. These cases are not at all easy to treat. Some seem to respond very well to vaccines; others do not respond at all.

My object is, however, to draw attention to the bladder as a possible cause of an attack of iritis, when no obvious cause of infection is to be found. Let me quote very briefly the notes of three cases:

(i) C. A. L., spinster, at. 45, consulted me in September, 1914. She gave a history that a fortnight previously one day she found she could only see one half of things at times. Just at first this phenomenon lasted a few minutes at a time, but later on in the day persisted almost without intermission. The following day the left eye was misty, and two days later it became bloodshot and painful. She had had a transient attack of "one-sided blindness" (hemianopia) followed by severe headaches when 15 years old, and had had two other attacks of inflammation in the right eye previous to the present attack. There was a history of slight constipation. When seen she had vision R.E. without glasses 6/12, with-0.25. D. sph., $0 \cdot 75$ D. cyl. axis $120^{\circ}=6 / 6$. L.E. $6 / 24$ and J 2 in each eye.

Examination.-In the L.E. there was no injection, but numerous fine spots on the lens capsule in the area of the pupil, and some vitreous opacities. The iris appeared normal.

She was wearing an artificial denture underneath which were several stumps. She had also a naso-pharyngeal discharge and much leucorrhœa: urine normal. She looked ill and had been losing weight for some time, and had suffered from stiff neck and pains in the shoulder, etc. All the stumps were removed from the mouth as soon as possible. The nasal sinuses were found to be clear and the naso-pharyngeal discharge soon improved under simple nasal douching. She was ordered to take small doses of Epsom salts daily, and the usual local treatment to the eye. In spite of this the left eye got worse, and at the end of a month the right eye showed marked signs of irido-cyclitis, and there were numerous "mutton-fat" deposits of keratitis punctata in each eye. In March, 1915, she was still doing badly and losing weight. Some crepitations were found in the right lung about that time, but no tubercle bacilli were found in the sputum. Injections of tuberculin were tried in small doses, gradually increasing in strength for some time, but with no effect upon her weight or the course of the iritis. In May, 1915, she was still losing weight rapidly ( $2 \frac{1}{2}$ lbs. in 14 days) and R.V. 6/60, L.V.6/18. No details of the fundi could be obtained, 
in either eye on account of the vitreous haze. Further examination at the end of June resulted in the isolation of bacillus coli from the urine and she was advised to have vaccines. This was started in July, 1915. After the sixth injection her sight was improved. In September, 1915, it was R.V.6/12, L.V. 6/18. There was keratitis punctata and vitreous opacities in both eyes still, but the fundus could be seen in the right eye and appeared normal. By October, 1915, her vision was R. 6/12 and L. 6/12 (some). The last injection was given at the end of October. Thereafter her general health improved, and she began to put on weight, but she continued to have transient attacks of pain and mistiness in one or the other eye, kept under control, however, by atropine. On the other hand, sight continued to improve steadily up to July, 1916, when she had vision R. 6/9 (ii), L. 6/6 (ii). There was no keratitis punctata to be seen in either eye, and just a few very fine opacities in the vitreous; fundi both normal.

(ii) The second case was that of E. E., spinster, at. 35, working as a tailoress, who was seen in August, 1915. She complained of mistiness of sight which had lasted six months, and had suffered from epilepsy for nine years. Her vision R. 6/12, J. 2, L. 6/18 (1) J. 2. She had a slight amount of mixed astigmatism, correction of which improved the sight of the left eye to 6/12. On examination under a mydriatic the pupils dilated well, but there was a large adhesion of left iris to lens capsule and in both eyes deposits on lens capsule and some fine keratitic deposits. Vitreous clear. Fundi normal. Her vision gradually got worse, but no cause for the irido-cyclitis could be found until November, when the urine was examined and found to yield pure cultures of bacillus coli. At that time her vision was R. 6/36, L. $6 / 60$; this was due to marked keratitis punctata in each eye and vitreous opacities. Autogenous vaccines were started, but she never responded to them, though her general condition was much improved. I lost sight of her for some months, but saw her again last March. Her condition was much the same, but her vision was R. 6/60 and L. $4 / 60$. At no time has there been any redness of the eye.

(iii) J. G. J., spinster, at. 18, seen September, 1915. She gave a history of constantly recurring frontal headaches for 12 months. Ten days before consulting me her eyes became inflamed; the left first and then the right. Her vision also began to get blurred. Her general health had been good. Vision : R. 6/12 and J. 2, L. 6/12 (1) and J. 2. No refractive error. Examination of R.E. showed marked keratitis punctata with normal pupil. Left eye showed slight ciliary haze, a large amount of keratitis punctata, and at one spot so thick was the lymph that it was hanging down in the anterior chamber from the back of the cornea, and there was a little hypopion. The pupil was sluggish, and new-formed blood- 
vessels were present on the surface of the iris. In both eyes some vitreous opacities were present. No obvious cause of sepsis was found until the bacillus coli was isolated in pure culture from the urine. She was at once given autogenous vaccines. In three weeks time there was no injection of the eyes; the keratitis punctata had practically disappeared and the vitreous seemed clear. I last saw her in November, 1915, i.e., two months after her first visit, and at that time her vision was R. 6/12, L. 6/9 (1). I understand she has remained well since.

I have had three or four other cases in which the bacillus coli has been found in pure culture from the urine, two of these were, I believe, in males, but I have not included those cases, as the investigation was not completed nor had special treatment been carried out.

There are just a few points with regard to the cases I have seen which I should like to dwell on.

Most of these cases have occurred in women and they have not uncommonly been associated with some constipation, though in the second and third cases this was not a prominent feature. But in view of the presence of constipation there is of course a flaw in the proof, for the infection may come from the alimentary tract primarily and not through the bladder, because of course the intestinal mucous membrane is more likely to be a good absorption area than that of the bladder. In view of that fact, I always prescribed daily doses of Epsom salts, but without influencing the disease until vaccines were given. The last case I quoted, however, is somewhat striking in the rapidity of the cure.

I have brought these cases forward, not because I think I have proved anything, but because it may possibly lead to other observations on the influence of unrecognized bladder infections as a cause of chronic inflammatory affections of the uveal tract. The colleague with whom I was working and who was doing the bacteriological work is at present in France, so that the work is now suspended, but I hope in happier days to come to resume this line of investigation.

\section{A CASE OF "UVEO-PAROTITIS WITH IRIDO-CYCLOPLEGIA."*}

BY

George Mackay, M.D., F.R.C.S.E. EDINBURGH.

ON March 10, 1916, Maggie R. (30), a tablemaid, was brought to me by her mistress. She looked well nourished, and there was

* A communication to the 1917 meeting of the Ophthalmological Society of the United Kingdom. The full text will appear in the Society's Transactions. 